\title{
Physical Activity For Multimorbidity Prevention And Management: A Systematic Review of Longitudinal Prospective Studies
}

\section{Danielle Hargan}

Queen's University Belfast

\section{Dáire McCartan}

Queen's University Belfast

Leandro M. T. Garcia ( $\sim$ L.Garcia@qub.ac.uk)

Queen's University Belfast

\section{Research Article}

Keywords: Physical activity, Multimorbidity, Chronic diseases, Non-communicable diseases, Review

Posted Date: June 2nd, 2021

DOI: https://doi.org/10.21203/rs.3.rs-537158/v1

License: (c) (i) This work is licensed under a Creative Commons Attribution 4.0 International License.

Read Full License 


\section{Abstract}

Background: The objective of this study was to determine whether physical activity can be used in the prevention and management of multimorbidity.

Methods: A systematic review was carried out using Pubmed, Web of Science, EMBASE, and Scopus databases to find experimental and prospective cohort studies that investigated the relationship between physical activity and prevention and management of multimorbidity. Participants consisted of general population, non-institutionalised adults aged 18 or more. Screening, data extraction, and risk of bias assessment were conducted by two independent reviewers.

Results: From a total of 1,724 studies identified, eight prospective cohort studies and one randomised control trial were included in the analysis. Four out of nine studies found evidence that higher levels of physical activity reduced the risk of multimorbidity. Three of four studies found evidence that physical activity reduces the risk of developing or worsening multimorbidity within subgroups with chronic conditions at the baseline.

Conclusion: The current evidence base indicates that it is unclear whether healthy individuals are more likely to develop multimorbidity if they are less physically active. However, it seems more likely for people to develop or worsen multimorbidity when they have one or any number of conditions at baseline if they are less physically active.

\section{Introduction}

Multimorbidity -the co-occurrence of two or more chronic illnesses in the same individual ${ }^{1}$-affects $95 \%$ of the population aged 65 and over; however, it does not exclusively affect the elderly. ${ }^{2}$ For instance, over 50 million people in Europe live with multimorbidity. ${ }^{2}$ Individuals who have a combination of chronic diseases are more likely to have lower quality of life, have more frequent hospital stays, and die prematurely. ${ }^{1,3}$ Multimorbidity also has a knock-on effect on an individual's mental wellbeing as it can lead to depression due to lack of physical functioning. ${ }^{4}$ Additionally, evidence suggests certain disease combinations increase the risk for certain health outcomes. ${ }^{4}$ For instance, people with combinations of congestive heart failure, diabetes, and chronic respiratory disease are more likely to have significant decline in functioning over four years. ${ }^{5}$

For health and social care systems, the costs of treating patients with multimorbidity is unsustainable and will have a negative impact in the quality of care on patients if new approaches are not considered. ${ }^{1,3}$ Patients with multimorbidity are more likely to be hospitalized for longer periods of time and the risk of excessive use of healthcare resources is much higher. $^{2}$

For many of the diseases contributing to multimorbidity, individual guidelines on prevention and management already exist. However, many of the issues faced by patients living with multimorbidity are 
not in the individual requirements of each disease, but rather in the interplay of the diseases. Prevention and management of multimorbidity, therefore, is difficult and requires a patient-centred model, focusing on multidisciplinary, integrated and coordinated care. ${ }^{2}$

Literature has vastly investigated the effects that physical activity has on preventing single chronic diseases, in improving quality of life of individuals, and preventing rapid progression of these diseases. However, only recently has literature begun to explore whether physical activity can have a role in multimorbidity prevention and management. One of the limitations of this knowledge lay in that there is not a systematic compilation of the literature to support the specific relationship between physical activity and multimorbidity, but rather on individual chronic diseases, which has been applied to multimorbidity. As multimorbidity is a growing area of public health concern, there is a requirement for in depth studies to determine whether physical activity can have a role in its prevention and management. Therefore, the aim of this study was to systematically review the existing longitudinal studies on the relationship between physical activity and multimorbidity prevention and management.

\section{Methods}

We conducted a systematic review of experimental and prospective cohort studies that investigated the relationship between physical activity practice (exposure) and incidence or management of multimorbidity (outcome). We included studies of general population, non-institutionalised adults aged 18 or more. Participants could either be healthy or already have chronic conditions at the beginning of the studies. No minimum intervention or follow-up period was considered. We excluded all studies with patients in health care settings (Table 1).

Physical activity could be in the domains of leisure, transport, household, and work, either alone or in combination. We did not restrict by type of instrument (self-report or device-based) or parameter of physical activity measured (e.g., weekly duration, frequency, or volume).

The outcome of this study was the occurrence of two or more chronic conditions (prevention) or the increment in the number of chronic conditions (management) at follow-up. This outcome was based on individuals who had no chronic conditions at baseline (prevention) and those with one or multiple chronic conditions at baseline (management). Multimorbidity was defined as per the studies and included various chronic conditions as those contributing to multimorbidity. 
Table 1. Inclusion and exclusion criteria according to population, exposure, comparator, outcomes, and study design of interest.

\section{INCLUSION CRITERIA}

\section{Population}

- Human adults aged 18 years or more

- Either healthy individuals (multimorbidity prevention) or individuals with chronic conditions (multimorbidity

management)

\section{Exposure}

- Physical activity practice at any level

- Occupational and/or non-occupational physical activity

- Self-reported or device-based measures

\section{Comparators}

- No physical activity or lower levels of physical activity

\section{Outcomes}

- Multimorbidity, as defined by studies

\section{Study design}

- Experimental studies or prospective cohorts

\section{EXCLUSION CRITERIA}

- Institutionalised adults (including in health care settings)
- Physical activity combined with other exposures

\subsection{Search strategy and sources}

A search for peer-reviewed academic papers was performed using Pubmed, Scopus, Web of Science, and EMBASE and the searches included studies from the inception of each database to 4 December 2019.

The search string used in each database can be found in the Additional File 1. The same terms were used in all the database searches and no language restrictions were applied. All the results from the database search were handled in the reference manager EndNote and all duplicates that could be found were removed. The articles were then uploaded to Covidence for the screening and selection process.

Two reviewers (DH, LG) independently screened titles and abstracts of all papers and then the full texts of the included articles. Conflicts were resolved via consensus in both stages. Reasons for exclusion during the full-text screening stage were provided according to the PECOS table (Table 1).

\subsection{Data extraction}

Two reviewers (DH, DM) independently extracted from each of the selected articles: author, publication year, study design, cohort/study name, country, sex and age range of participants, definition of 
multimorbidity used, physical activity measurement method or intervention protocol, physical activity domains, number of participants and cases of multimorbidity per physical activity category, and risk estimates. Conflicts were resolved via consensus.

\subsection{Risk of bias assessment}

Two reviewers (DH, DM) independently assessed the risk of bias of the selected papers. The NewcastleOttawa Quality Assessment Scale for Cohort Studies ${ }^{6}$ and the US National Heart, Lung, and Blood Institute's Scale for Controlled Intervention Studies ${ }^{7}$ were used to evaluate the quality of prospective cohorts and experimental studies, respectively. Conflicts between reviewers were resolved via consensus.

\section{Results}

The overall number of studies that were identified as potentially relevant from the database searches was 1,724 , of which 857 were duplicates and 867 progressed to title and abstract screening. A total of 82 articles were retrieved for full-text screening. Nine papers ${ }^{8-16}$ were selected to be part of this review (Fig. 1).

Table S1 (Additional file 2) presents the main characteristics and findings of the nine selected papers. The first study was published in 2015 and the remaining ones were published between 2017 and 2019. One of the studies was a randomised control trial and the others were prospective cohorts. The articles included were all in English and were conducted in Canada, Scotland, England, Ireland, Finland, and Australia.

The participants in most of the articles were middle-aged. Four included participants who were aged 50 and above, ${ }^{11-14}$ one involved participants aged $45-50,{ }^{16}$ and one had participants aged $60-77 .{ }^{10}$ Three availed of data from both young and middle-aged adults, with age ranges of $18-75,20-69$ and 25$64 . .^{8,9,15}$ One study only reported results for women ${ }^{16}$ and another provided separate results for men and women with different baseline chronic conditions, ${ }^{15}$ whereas the rest of the studies included combined results for both men and women.

All eight cohort studies $8,9,11-16$ availed of self-reported methods to collect data on the level of physical activity of participants. Follow-up length ranged from 10 to 32 years, except for one study with a 2-year follow-up. ${ }^{12}$

One study was a randomised control trial. ${ }^{10}$ The intervention group received an intensive multidomain intervention for two years. For the physical activity aspect, participants in the intervention group received training that was guided by physical therapists using individually tailored programs. They used a modified version of the Dose Response to Exercise Training (DR EXTRA) study protocol. Participants received muscle strength training 1-3 times per week, aerobic exercise 2-5 times per week and posture improving exercises 1-3 times per week, as well as receiving nutritional guidance and cognitive training. 
However, the control group received regular general health advice. The groups were allocated randomly using computer-generated allocation.

Some of the studies provided evidence on subgroups of those with multimorbidity depending on baseline characteristics, such as the number of conditions at baseline, ${ }^{10-12}$ or the type of condition participants had been diagnosed prior to study. ${ }^{15}$ The number and type of chronic conditions included varied between studies. Some of the studies chose to limit the number of conditions or groups of conditions that were included, whereas one study had up to 40 conditions. Illnesses were measured by self-reporting the conditions through questionnaires or through data retrieved from medical records.

\subsection{Risk of bias assessment}

The eight prospective cohort studies were assessed using the Newcastle-Ottawa Quality Assessment Scale for Cohort Studies ${ }^{6}$ (Additional File 3, Table S2). Three studies ${ }^{8,9,13}$ scored six, three ${ }^{11,12,16}$ scored seven, and two ${ }^{14,15}$ scored eight out of nine points. The majority of the studies failed to have a follow-up rate of at least $80 \%$, with just three of the eight studies achieving that.

The randomised control trial ${ }^{10}$ was assessed using the US National Heart, Lung, and Blood Institute's Scale for Controlled Intervention Studies, ${ }^{7}$ and scored 12 out of 14 points, with little risk of bias (Additional File 3, Table S3).

\subsection{Relationship between physical activity and multimorbidity prevention and management}

All nine studies reported results of incident multimorbidity for healthy participants at baseline $(11-19) .^{8-}$

${ }^{16}$ No evidence of relationship between physical activity and multimorbidity was observed in five of these studies, ${ }^{8-10,12,16}$ however, other four found an increase in risk of multimorbidity among those less physically active. ${ }^{11,13-15}$ In the study by Singer et al., ${ }^{13}$ the risk of incident multimorbidity from doing moderate physical activity compared with vigorous physical activity was 1.22 (95\% confidence interval (Cl): 1.16-1.28), increasing to 1.57 (95\% Cl: 1.46-1.68) and 1.60 (95\% Cl: $1.45-1.76)$ for those doing mild or no physical activity, respectively. In the study by Singh-Manoux et al., ${ }^{14}$ participants who engaged in less than 2.5 hours/week of moderate or vigorous physical activity were more likely to have multimorbidity at follow-up than those above the threshold $(1.21,95 \% \mathrm{Cl}: 1.01-1.45)$. In the study by Mounce et al., ${ }^{11}$ those in the low/sedentary category were more likely to have multimorbidity at follow-up than those who were highly active $(1.43,95 \% \mathrm{Cl}: 1.02-2.00)$. Wikström et al. ${ }^{15}$ found that me initially disease-free and who took part in low levels of physical activity at baseline were more likely to have multimorbidity at follow-up than those who were highly active $(1.34,95 \% \mathrm{Cl}: 1.03-1.73)$, as were women (1.62, 95\% Cl: 1.14-2.30).

Additionally, four studies investigated the risk of developing or worsening multimorbidity within subgroups with chronic conditions at the baseline. ${ }^{10-12,15}$ In the randomised control trial conducted by 
Marengoni et al., ${ }^{10}$ participants in the intervention group who had one or more chronic diseases at baseline had a lower risk of developing or worsening multimorbidity at follow-up compared to the control group $(0.80,95 \% \mathrm{Cl}: 0.64-0.99)$. In the study by Mounce et al., ${ }^{11}$ those with any number of chronic conditions at baseline who were in the low/sedentary category were more likely to developing or worsening multimorbidity at follow-up than those in who were highly active $(1.19,95 \% \mathrm{Cl}$ : 1.06-1.35). Wikström et al. ${ }^{15}$ found that men with diabetes at baseline and who took part in low levels of physical activity had greater risk of multimorbidity in comparison to those that reported high levels of physical activity $(1.80,95 \% \mathrm{Cl}: 1.29-2.53)$. However, they did not find evidence of association among women with diabetes and men and women with cardiovascular diseases at baseline. One other study did not find evidence of developing or worsening multimorbidity according to physical activity behaviour or people with chronic conditions at baseline. ${ }^{12}$

\section{Discussion}

The findings of this systematic review suggest that the effectiveness of physical activity in preventing the onset of multimorbidity is still unclear, as is its role in the management of multimorbidity. The conflicting results that were apparent from the systematic review and from previous literature would suggest that it remains difficult to distinguish whether physical activity on its own can act as an effective intervention for preventing and managing multimorbidity. However, it is possible that individuals may be more likely to develop multimorbidity if they are less physically active, particularly if they are in the sedentary/low level category. It seems more likely for participants to develop or worsen multimorbidity when they have one or any number of conditions at baseline if they are in the low physical activity level category than if they are in the high/vigorous physical activity level category.

Other studies investigating the relationship between physical activity and multimorbidity have suggested that, due to the nature of some of the conditions associated with multimorbidity and their symptoms, engagement in regular physical activity can be reduced. Dankel et al. ${ }^{17}$ found that individuals with multimorbidity are less likely to engage in muscle-strengthening activities and physical activity in general because of their diseased state. A reason for this can be seen in the study by Dörenkamp et al., ${ }^{18}$ who found that individuals with multiple different conditions, including chronic respiratory disease, lower back pain, inflammatory joint disease, among others, experienced high levels of pain which, therefore, decrease their functional ability and thereby interfere with participation in adequate physical activity levels. Ryan et al. ${ }^{19}$ also report that multimorbidity can result in future functional decline, therefore individuals with poorer physical function may be less inclined or able to participate in physical activity.

Furthermore, our results might indicate that physical activity on its own may not be a major factor in multimorbidity prevention and management, but rather should be combined with other health behaviourrelated factors such as better diet, lower body mass index, low alcohol consumption, etc. This is supported in the study by Fortin et al., ${ }^{20}$ who found that an accumulation of unhealthy lifestyle factors was likely to increase multimorbidity. 
It is important to note that providing preventive and treatment services to individuals with multimorbidity requires a patient and disease-combination specific approach rather than a one-size-fits-all model.

Pearson-Stuttard et al. ${ }^{21}$ recommend that the best way to approach multimorbidity requires interventions at individual and populations levels in conjunction with healthcare, public health, and social-care policies. Barnett et al. ${ }^{22}$ state that clinical evidence and guidelines are largely created for individual diseases and suggest that trials using more representative populations are needed to add to existing evidence. Both these recommendations would likely lead to accelerated progress within the clinical setting at improving the prevention and treatment services, therefore improving the level of care provided and increasing quality of life of individuals.

\subsection{Strengths and limitations}

One of the main limitations of the study was in measuring multimorbidity, due to a lack of a uniform number and types of chronic conditions across studies. Some of the studies included in the review used a smaller number of conditions or used domains of conditions to assess multimorbidity, whereas other studies used a larger variety of conditions. This was largely down to the authors of the studies tailoring the measure of multimorbidity to match the focus of the study. The importance of publishing a list of conditions that contribute to multimorbidity is highlighted by Stewart et al. ${ }^{23}$ The UK National Institute for Health and Care Excellence's guidelines provide a list of domains that cover a variety of physical and mental health conditions, ongoing conditions such as learning disabilities and sensory impairments, and more. ${ }^{24}$ However, this is an overview of an extensive list of conditions and does not provide a clear focus on the types of conditions that should be considered as contributing to multimorbidity. This too suggests that measuring multimorbidity is study specific. The issue that arises with non-uniform definition and measurement is that the prevalence of multimorbidity may be over- or under-estimated. Also, the relevance of physical activity in the prevention and management of individual chronic conditions used to define multimorbidity across studies varies. Therefore, the results of the studies may be affected. ${ }^{23,25}$ These sources of heterogeneity also preclude a meaningful meta-analysis of the evidence available so far.

Another limitation in this study was the way physical activity was measured and recorded. All studies included in the review used different self-reported methods to ascertain the levels of physical activity, affecting the reliability of the physical activity measures and raising issues for comparing the studies in the review. Moreover, older adults-the target group of many of the selected studies-tend to over-report their physical activity, and vigorous activities are likely to be recalled more accurately than non-vigorous activities. $^{26}$

Strengths of the systematic review lie largely in the protocol that was followed, reducing bias when carrying out the stages of the review, such as running the searches, using multiple databases as sources, and two independent reviewers in all stages. A comprehensive list of search terms was used which enabled us to find of a vast amount of relevant literature. All studies included in the review had relatively 
large sample size and none had high risk of biases. Finally, this study relied only on longitudinal designs, mitigating at least some of potential reverse causality issues.

\section{Conclusion}

There has been conflicting evidence on the sole use of physical activity for the prevention and management of multimorbidity. However, potential benefits cannot be ruled out based on the evidence currently available. As a growing area of public health concern, it requires further in-depth studies, including well-designed trials, into whether physical activity and its multitude of both short- and long-term health benefits could have a significant positive role to play. Including more individuals with multimorbidity in relevant clinical trials may also lead to further discoveries of the benefits of physical activity on the management of multiple chronic conditions.

\section{Declarations}

Ethics approval and consent to participate

Not applicable.

Consent for publication

Not applicable.

Availability of data and materials

All data generated or analysed during this study are included in this published article and its supplementary information files.

Competing interests

The authors declare that they have no competing interests.

\section{Funding}

This research did not receive any specific grant from funding agencies in the public, commercial, or notfor-profit sectors.

\section{Authors' contributions}

DH and LG designed the work and screened and selected the studies. DH and DM extracted the data and conducted the risk of bias assessment. DH drafted the manuscript. DM and LG substantively revised the manuscript. All authors read and approved the final manuscript. 
We would like to thank Dr Richard Fallis, subject librarian, who provided guidance on literature searches and how to use the various databases.

\section{References}

1. MacMahon S. Multimorbidity: a priority for global health research. London: The Academy of Medical Sciences; 2018.

2. Navickas R, Petric VK, Feigl AB, Seychell M. Multimorbidity: What do we know? What should we do? J Comorb. 2016;6(1):4-11.

3. Mercer S, Furler J, Moffat K, Fischbacher-Smith D, Sanci L. Multimorbidity: technical series on safer primary care. Geneva: World Health Organization; 2016.

4. Xu X, Mishra GD, Jones M. Evidence on multimorbidity from definition to intervention: an overview of systematic reviews. Ageing Res Rev. 2017;37:53-68.

5. Bayliss EA, Bayliss MS, Ware JE, Jr., Steiner JF. Predicting declines in physical function in persons with multiple chronic medical conditions: what we can learn from the medical problem list. Health Qual Life Outcomes. 2004;2:47.

6. Wells G, Shea B, O'Connell D, Peterson J, Welch V, Losos M, et al. The Newcastle-Ottawa Scale (NOS) for assessing the quality of nonrandomised studies in meta-analyses [Accessed 11 Nov 2020]. Available from: http://www.ohri.ca/programs/clinical_epidemiology/oxford.asp.

7. U.S. National Heart, Lung, and Blood Institute. Quality assessment of controlled intervention studies [Accessed 11 Nov 2020]. Available from: https://www.nhlbi.nih.gov/health-topics/study-qualityassessment-tools.

8. Canizares M, Hogg-Johnson S, Gignac MAM, Glazier RH, Badley EM. Increasing trajectories of multimorbidity over time: birth cohort differences and the role of changes in obesity and income. $J$ Gerontol B Psychol Sci Soc Sci. 2018;73(7):1303-14.

9. Katikireddi SV, Skivington K, Leyland AH, Hunt K, Mercer SW. The contribution of risk factors to socioeconomic inequalities in multimorbidity across the lifecourse: a longitudinal analysis of the Twenty-07 cohort. BMC Med. 2017;15(1):152.

10. Marengoni A, Rizzuto D, Fratiglioni L, Antikainen R, Laatikainen T, Lehtisalo J, et al. The effect of a 2year intervention consisting of diet, physical exercise, cognitive training, and monitoring of vascular risk on chronic morbidity - the FINGER randomized controlled trial. J Am Med Dir Assoc. 2018;19(4):355-60.e1.

11. Mounce LTA, Campbell JL, Henley WE, Tejerina Arreal MC, Porter I, Valderas JM. Predicting incident multimorbidity. Ann Fam Med. 2018;16(4):322-9.

12. Ryan A, Murphy C, Boland F, Galvin R, Smith SM. What is the impact of physical activity and physical function on the development of multimorbidity in older adults over time? A population-based cohort study. J Gerontol A Biol Sci Med Sci. 2018;73(11):1538-44. 
13. Singer L, Green M, Rowe F, Ben-Shlomo Y, Morrissey K. Social determinants of multimorbidity and multiple functional limitations among the ageing population of England, 2002-2015. SSM Popul Health. 2019;8:100413.

14. Singh-Manoux A, Fayosse A, Sabia S, Tabak A, Shipley M, Dugravot A, et al. Clinical, socioeconomic, and behavioural factors at age 50 years and risk of cardiometabolic multimorbidity and mortality: a cohort study. PLoS Med. 2018;15(5):e1002571.

15. Wikström K, Lindström J, Harald K, Peltonen M, Laatikainen T. Clinical and lifestyle-related risk factors for incident multimorbidity: 10-year follow-up of Finnish population-based cohorts 19822012. Eur J Intern Med. 2015;26(3):211-6.

16. Xu X, Mishra GD, Dobson AJ, Jones M. Progression of diabetes, heart disease, and stroke multimorbidity in middle-aged women: a 20-year cohort study. PLoS Med. 2018;15(3):e1002516.

17. Dankel SJ, Loenneke JP, Loprinzi PD. Combined associations of muscle-strengthening activities and accelerometer-assessed physical activity on multimorbidity: findings from NHANES. Am J Health Promot. 2017;31(4):274-7.

18. Dörenkamp S, Mesters I, Vos R, Schepers J, van den Akker M, Teijink J, et al. Synergistic effects of six chronic disease pairs on decreased physical activity: the SMILE Cohort Study. Biomed Res Int. 2016;2016:9427231.

19. Ryan A, Wallace E, O'Hara P, Smith SM. Multimorbidity and functional decline in community-dwelling adults: a systematic review. Health Qual Life Outcomes. 2015;13:168.

20. Fortin M, Haggerty J, Almirall J, Bouhali T, Sasseville M, Lemieux M. Lifestyle factors and multimorbidity: a cross sectional study. BMC Public Health. 2014;14:686.

21. Pearson-Stuttard J, Ezzati M, Gregg EW. Multimorbidity - a defining challenge for health systems. Lancet Public Health. 2019;4(12):e599-e600.

22. Barnett K, Mercer SW, Norbury M, Watt G, Wyke S, Guthrie B. Epidemiology of multimorbidity and implications for health care, research, and medical education: a cross-sectional study. Lancet. 2012;380(9836):37-43.

23. Stewart M, Fortin M, Britt HC, Harrison CM, Maddocks HL. Comparisons of multi-morbidity in family practice - issues and biases. Fam Pract. 2013;30(4):473-80.

24. U.K. National Institute for Health and Care Excellence. Overview: Multimorbidity - clinical assessment and management. London: U.K. National Institute for Health and Care Excellence; 2016.

25. Johnston MC, Crilly M, Black C, Prescott GJ, Mercer SW. Defining and measuring multimorbidity: a systematic review of systematic reviews. Eur J Public Health. 2019;29(1):182-9.

26. Dhalwani NN, O'Donovan G, Zaccardi F, Hamer M, Yates T, Davies M, et al. Long terms trends of multimorbidity and association with physical activity in older English population. Int J Behav Nutr Phys Act. 2016;13:8.

\section{Figures}




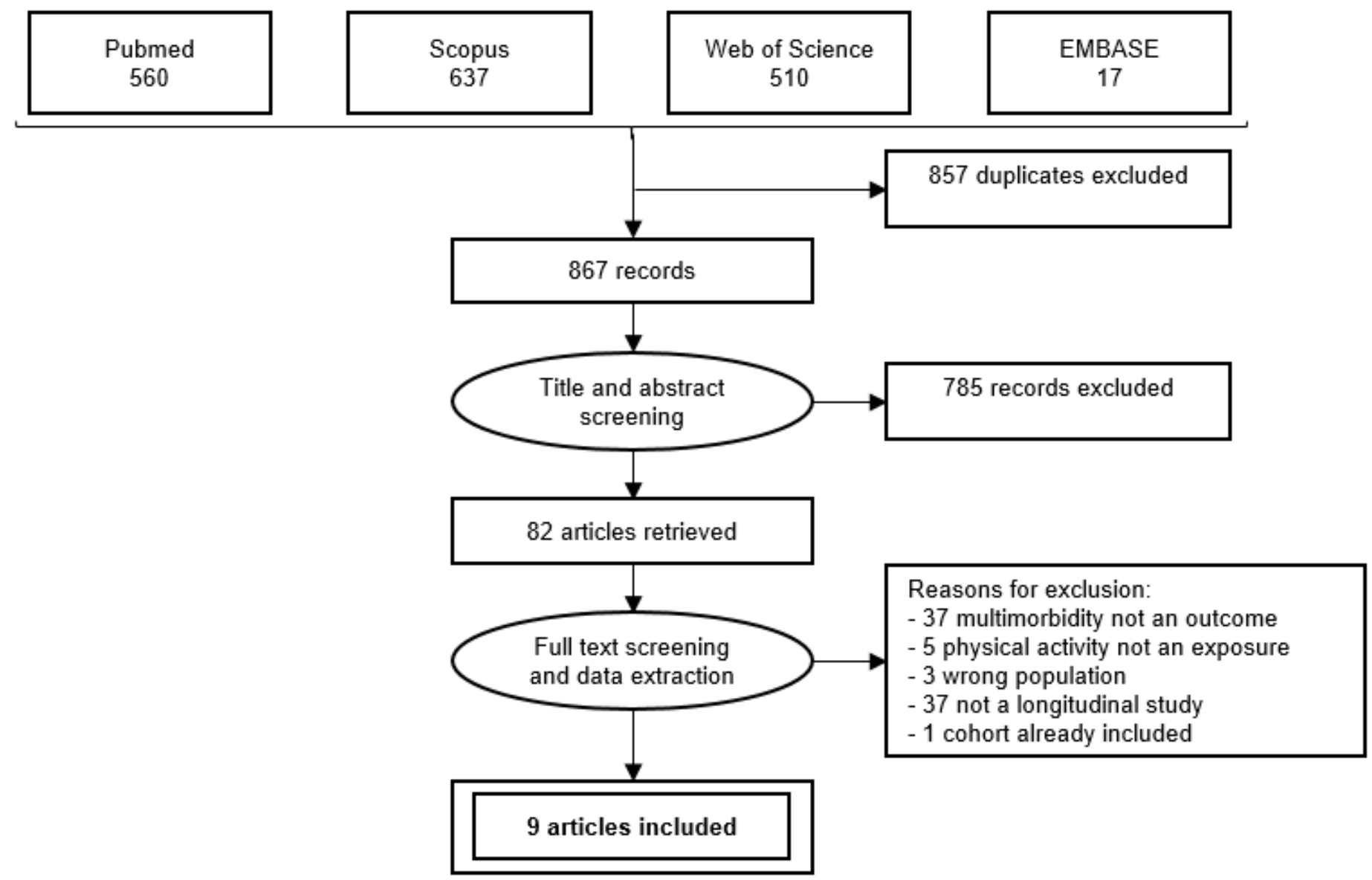

Figure 1

Search and selection process.

\section{Supplementary Files}

This is a list of supplementary files associated with this preprint. Click to download.

- Additionalfile1.docx

- Additionalfile2.xlsx

- Additionalfile3.docx 